\title{
Automatic Memory Access Analysis with Periscope
}

\author{
Michael Gerndt and Edmond Kereku \\ Technische Universität München \\ Fakultät für Informatik I10, Boltzmannstr. 3, 85748 Garching \\ gerndt@in.tum.de
}

\begin{abstract}
Periscope is a distributed automatic online performance analysis system for large scale parallel systems. It consists of a set of analysis agents distributed on the parallel machine. This article presents the support in Periscope for analyzing inefficiencies in the memory access behavior of the applications. It applies data structure specific analysis and is able to identify performance bottlenecks due to remote memory access on the Altix 4700 ccNUMA supercomputer.
\end{abstract}

Keywords: Performance analysis, supercomputers, program tuning, memory accesses analysis.

\section{Introduction}

Performance analysis tools help users in writing efficient codes for current high performance machines. Since the architectures of today's supercomputers with thousands of processors expose multiple hierarchical levels to the programmer, program optimization cannot be performed without experimentation.

To tune applications, the user has to carefully balance the number of MPI processes vs the number of threads in a hybrid programming style, he has to distribute the data appropriately among the memories of the processors, has to optimize remote data accesses via message aggregation, prefetching, and asynchronous communication, and, finally, has to tune the performance of a single processor.

Performance analysis tools can provide the user with measurements of the the program's performance and thus can help him in finding the right transformations for performance improvement. Since measuring performance data and storing those data for further analysis in most tools is not a very scalable approach, most tools are limited to experiments on a small number of processors. To investigate the performance of large experiments, performance analysis has to be done online in a distributed fashion, eliminating the need to transport huge amounts of performance data through the parallel machine's network and to store those data in files for further analysis.

Periscope [5] is such a distributed online performance analysis tool. It consists of a set of autonomous agents that search for performance bottlenecks in a 
subset of the application's processes and threads. The agents request measurements of the monitoring system, retrieve the data, and use the data to identify performance bottlenecks. The types of bottlenecks searched are formally defined in the APART Specification Language (ASL) [12].

The focus of this paper is on Periscope's support for analyzing the memory access behavior of programs. Periscope searches not only for bottlenecks related to MPI and OpenMP, but also for inefficiencies in the memory accesses. Novel features are the support for identifying data structure-related bottlenecks and remote memory accesses bottlenecks in ccNUMA systems.

The next section presents work related to the automatic performance analysis approach in Periscope. Section 3 presents Periscope's architecture and its special features for memory access analysis. Section [5 presents several case studies. Section 6 gives a short summary.

\section{Related Work}

Several projects in the performance tools community are concerned with the automation of the performance analysis process. Paradyn's 9] Performance Consultant automatically searches for performance bottlenecks in a running application by using a dynamic instrumentation approach. Based on hypotheses about potential performance problems, measurement probes are inserted into the running program. Recently MRNet [10] has been developed for the efficient collection of distributed performance data.

The Expert 12 tool developed at Forschungszentrum Jülich performs an automated post-mortem search for patterns of inefficient program execution in event traces. Potential problems with this approach are large data sets and long analysis times for long-running applications that hinder the application of this approach on larger parallel machines.

Aksum [3], developed at the University of Vienna, is based on a source code instrumentation to capture profile-based performance data which is stored in a relational database. The data is then analyzed by a tool implemented in Java that performs an automatic search for performance problems based on JavaPSL, a Java version of ASL.

\section{Architecture}

Periscope consists of a graphical user interface based on Eclipse, a hierarchy of analysis agents and two separate monitoring systems (Figure 11).

The graphical user interface allows the user to start up the analysis process and to inspect the results. The agent hierarchy performs the actual analysis. The node agents autonomously search for performance problems which have been specified with ASL. Typically, a node agent is started on each SMP node of the target machine. This node agent is responsible for the processes and threads on that node. Detected performance problems are reported to the master agent that communicates with the performance cockpit. 


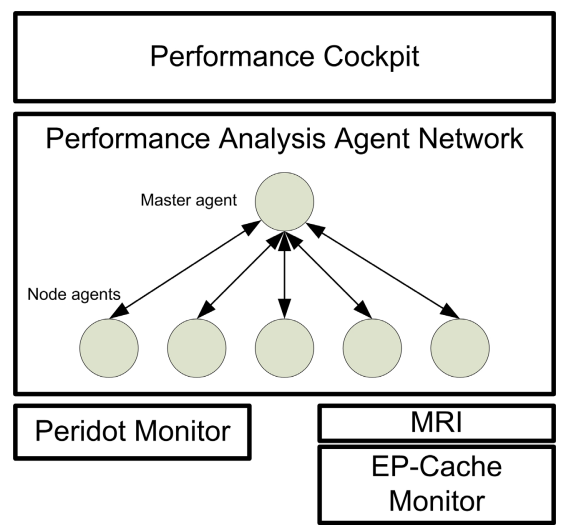

Fig. 1. Periscope currently consists of a GUI based on Eclipse, a hierarchy of analysis agents, and two separate monitoring systems

The node agents access a performance monitoring system for obtaining the performance data required for the analysis. Periscope currently supports two different monitors, the Peridot monitor 4 developed in the Peridot project focusing on OpenMP and MPI performance data, and the EP-Cache monitor 8 developed in the EP-Cache project focusing on memory hierarchy information.

The node agents perform a sequence of experiments. Each experiment lasts for a program phase, which is defined by the programmer, or for a predefined amount of execution time. Before a new experiment starts, an agent determines a new set of hypothetical performance problems based on the predefined ASL properties and the already found problems. It then requests the necessary performance data for proving the hypotheses and starts the experiment. After the experiment, the hypotheses are evaluated based on the performance data obtained from the monitor.

\section{Monitoring Memory Accesses}

The analysis of the application's memory access behavior is based on the EPCache monitor. It allows to access the hardware counters of the machine for evaluating properties such as the example for L1-cache misses in Figure 2

The example demonstrates the specification of performance properties with ASL. The performance property shown here identifies a region with high L1cache miss rate. The data model, specified in ASL too, contains a class SeqPerf which contains a reference to a program region, a reference to a process, and a number of cache-related and other metrics. The instance of SeqPerf available in the property is called the property's context. It defines the program region, e.g., a function, and the process for which the property is tested.

A novel feature of the EP-Cache monitor is its data structure support. The node agent can request measurements for specific data structures via the 


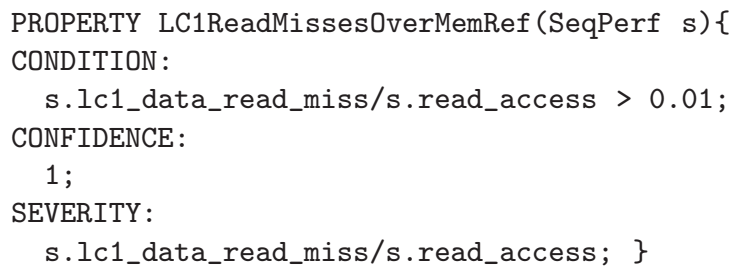

Fig. 2. This performance property identifies significant L1-cache miss rate

Measurement Request Interface (MRI) [6]. The node agent specifies a request via the MRI_Request function. Its parameters determine, for example, that L1cache misses are to be measured for array ACOORD in a specific parallel loop in subroutine FOO. The request would be generated for a new experiment after the property LC1MissRate was proven in the previous experiment.

To enable the node agent to specify such detailed requests, it requires static information about the program, e.g., it has to know that array ACOORD is accessed in that loop. This program information is generated by the source-tosource instrumenter developed for Periscope. It generates the information in an XML-based format called the Standard Intermediate Program Representation (SIR) [1]. This information is read by the agents when the analysis is started.

At runtime the MRI request for measuring cache misses for a data structure is translated based on the debug information in the executable into the associated range of virtual addresses. The measurement is certainly only possible, if the hardware counters of the target processor do support such restricted counting. Our main target architecture is the Altix 4700, which was installed at the Leibniz Computing Centre in Munich. It consists of 4096 Itanium 2 processors which have a very extensive set of hardware counters. On Itanium 2, such measurements can be restricted to specific address ranges and thus can be executed without program intrusion.

Another feature of the Itanium 2's hardware counters exploited by Periscope is its support for counting memory accesses that last more than a given number of clock cycles. One of these events is DATA_EAR_CACHE_LAT8 for example. This event returns the number of memory operations with a memory access latency of more than eight cycles. Similar events return the number of operations with a memory access latency greater than LAT4, LAT16, ... up to LAT4096 increasing by powers of 2 .

The counters can be used to identify those memory references that go to local memory on the ALTIX 4700 processor or to remote memory. Since the ALTIX is a ccNUMA system, all the processors can access all the memory, but, for efficieny reasons, most of the accesses should be to the processors local memory. Thus it is very important to identify non-local access behavior. Edmond Kereku specified appropriate ASL performance properties for such situation in his dissertation [7].

Based on elementary properties such as the LC1Miss-Property and similar properties for individual data structures and for remote memory accesses, 
higher-level properties can be deduced. For example, the Property UnbalancedDMissRateInThreads shown in Figure 3 gives information on the behavior across multiple threads.

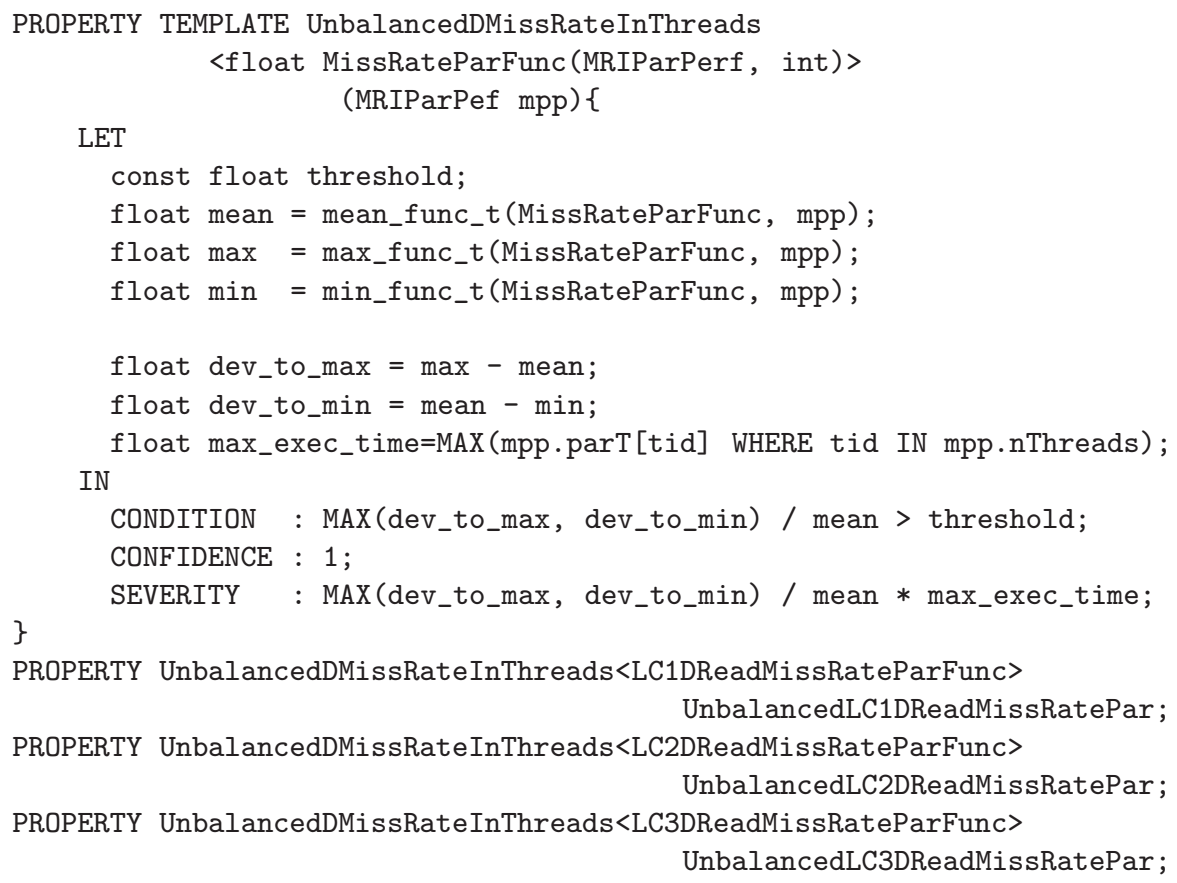

Fig. 3. Performance properties with a similar specification can be expressed via property templates. This template specified an unbalanced miss rate across threads in a parallel OpenMP program.

The template UnbalancedDMissRateInThreads is used to create multiple properties for the different cache levels. The property template is parameterized by a function which is replaced in the specifications of the individual properties with a function returning the appropriate miss rate, i.e., for the L1 cache, L2, and L3 respectively.

\section{Application Experiments}

We used Periscope to analyze applications on the Itanium SMP nodes of the Infiniband Cluster at LRR/TUM. Table1 shows the list of properties which we searched for during our experiments, the threshold set in the condition of the properties, and the number of regions on which the property holds for each of the 
Table 1. The list of properties searched on the test applications running on the Infiniband Cluster

\begin{tabular}{|c|r|r|r|r|r|}
\hline Property & Threshold & LU & FT & SWIM & PGAUSS \\
\hline \hline LC2DMissRate & $5 \%$ & 0 & 14 & 0 & 5 \\
LC2DReadMissRate & $5 \%$ & 0 & 3 & 0 & 5 \\
LC3DMissRate & $2 \%$ & 13 & 3 & 7 & 0 \\
LC3DReadMissRate & $2 \%$ & 1 & 3 & 2 & 0 \\
\hline
\end{tabular}

test applications. We did not count the data structures, only the code regions. The properties hold for a region if the cache miss rate is higher than $5 \%$ for LC2 and higher than $2 \%$ for LC3. Looking at the table, we can conclude that FT and PGAUSS have more L2 cache problems, while LU and SWIM have more L3 cache problems.

We present here the results of Periscope's automatic search for the LU decomposition application in the form of search paths. The paths start from the region where the search began and go down to the deepest subregion or data structure for which the property holds. We omit the severities associated with each evaluated property. Instead, we provide the measured miss rate for each of the search path's regions and data structures. The search path which generated the property with the highest severity is marked italic. Please note that in some of the tests, the region with the highest miss rate does not necessarily have the property with the highest severity. The severity is also dependant on the region's execution time, which for presentation reasons is not shown here.

The results of the automatic search for $L U$

Region

Application Phase( USER REGION, ssor.f, 109 )

( PARALLEL REGION, ssor.f, 120 )

( WORKSHARE DO, ssor.f, 126 ) rsd( DATA STRUCTURE, ssor.f, 4 )

Application Phase( USER REGION, ssor.f, 109 )

( PARALLEL REGION, ssor.f, 120 )

( LOOP REGION, ssor.f, 149 )

jacld( CALL REGION, ssor.f, 156 )

jacld( SUB REGION, jacld.f, 5 )

( WORKSHARE DO, jacld.f, 39 ) u( DATA STRUCTURE, jacld.f, 5)

\section{LC3 miss}

rate

0.038

0.029

0.029

0.038

0.037

0.030

0.030

0.040

Application Phase( USER REGION, ssor.f, 109 )

( PARALLEL REGION, ssor.f, 120 )

0.038

( LOOP REGION, ssor.f, 149 )

0.037

blts ( CALL REGION, ssor.f, 165 )

0.052

blts ( SUB REGION, blts.f, 4 ) 

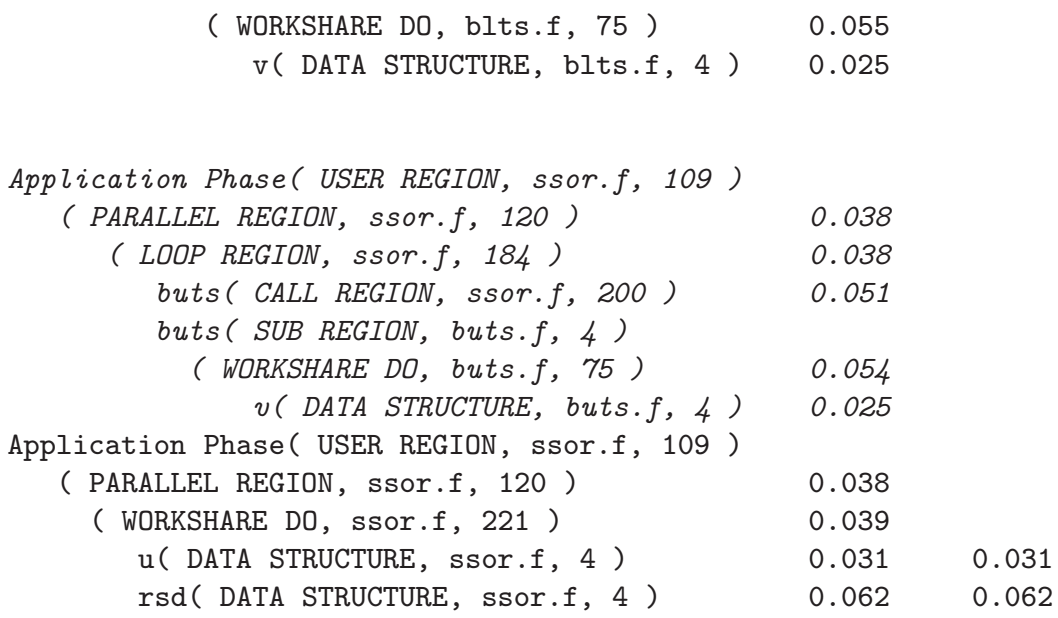

The search for L2 cache problems did not detect any problem, but as the results show, LU has L3 cache problems. In addition to L3DMissRate, our search refined to the property L3DReadMissRate too. As the search paths show, LC3DReadMissRate does not hold on the majority of the regions where LC3MissRate was proven. This means that most of the L3 cache problems in LU are write-related problems.

The search path that discovered the most severe problem refined from the application phase to subroutine buts. The data structure $\mathrm{v}$ is the source of the problem. The most important data structures of LU are $u$ and rsd. In fact, the variable $\mathrm{v}$ is the local name for $\mathrm{rsd}$ which is passed to subroutine buts as a parameter.

\section{Summary}

This paper presented the automatic memory access analysis support in Periscope. Periscope's analysis is automatically running several experiments during a single program execution to incrementally search for performance bottlenecks. If no repetitive program phases are marked by the user, the application can even be automatically restarted to perform additional experiments.

Periscope uses this approach to search for data structure-related memory access bottlenecks as well as for remote memory access bottlenecks in ccNUMA architectures. Due to the limitation in the number of performance counters of current processors, multiple experiments are required to evaluate all the performance hypotheses for critical program regions.

The overall overhead of the analysis depends on the frequency measurements are taken. In our tests, the regions to be analyzed where outer loops or parallel regions which have significant runtime so that accesses to the performance counters did not have significant overhead. 
The strategies applied in refining for more detailed performance bottlenecks can be found in [7] and will be published elsewhere.

Acknowledgments. This work is funded by the Deutsche Forschungsgemeinschaft under Contract No. GE 1635/1-1.

\section{References}

1. T. Fahringer, M. Gerndt, G. Riley, and J. Träff. Knowledge specification for automatic performance analysis. APART Technical Report, www.fz-juelich.de/apart, 2001.

2. T. Fahringer, M. Gerndt, G. Riley, and J.L. Träff. Specification of performance problems in MPI-programs with ASL. International Conference on Parallel Processing (ICPP'00), pp. 51 - 58, 2000.

3. T. Fahringer and C. Seragiotto. Aksum: A performance analysis tool for parallel and distributed applications. Performance Analysis and Grid Computing, Eds. V. Getov, M. Gerndt, A. Hoisie, A. Malony, B. Miller, Kluwer Academic Publisher, ISBN 1-4020-7693-2, pp. 189-210, 2003.

4. K. Fürlinger and M. Gerndt. Peridot: Towards automated runtime detection of performance bottlenecks. High Performance Computing in Science and Engineering, Garching 2004, pp. 193-202, Springer, 2005.

5. M. Gerndt, K. Fürlinger, and E. Kereku. Advanced techniques for performance analysis. Parallel Computing: CurrentEFFure Issues of High-End Computing (Proceedings of the International Conference ParCo 2005), Eds: G.R. Joubert, W.E. Nagel, F.J. Peters, O. Plata, P. Tirado, E. Zapata, NIC Series Volume 33 ISBN 3-00-017352-8, pp. 15-26a, 2006.

6. M. Gerndt and E. Kereku. Monitoring Request Interface version 1.0. TUM Technical Report, 2003.

7. E. Kereku. Automatic Performance Analysis for Memory Hierarchies and Threaded Applications on SMP Systems. PhD thesis, Technische Universität München, 2006.

8. E. Kereku and M. Gerndt. The EP-Cache automatic monitoring system. International Conference on Parallel and Distributed Systems (PDCS 2005), 2005.

9. B.P. Miller, M.D. Callaghan, J.M. Cargille, J.K. Hollingsworth, R.B. Irvin, K.L. Karavanic, K. Kunchithapadam, and T. Newhall. The Paradyn parallel performance measurement tool. IEEE Computer, Vol. 28, No. 11, pp. 37-46, 1995.

10. Ph. C. Roth, D. C. Arnold, and B. P. Miller. MRNet: A software-based multicast/reduction network for scalable tools. SC2003, Phoenix, November, 2003.

11. C. Seragiotto, H. Truong, T. Fahringer, B. Mohr, M. Gerndt, and T. Li. Standardized Intermediate Representation for Fortran, Java, C and $\mathrm{C}++$ programs. APART Working Group Technical Report, Institute for Software Science, University of Vienna, Octorber, 2004.

12. F. Wolf and B. Mohr. Automatic performance analysis of hybrid MPI/OpenMP applications. 11th Euromicro Conference on Parallel, Distributed and NetworkBased Processing, pp. 13 - 22, 2003. 